DOI: $10.17516 / 1997-1397-2021-14-3-369-375$

УДК 517.55

\title{
Removable Singularities of Separately Harmonic Functions
}

\author{
Sevdiyor A. Imomkulov* \\ Khorezm Regional Branch of the V. I. Romanovsky Mathematical Institute \\ Academy of Sciences of the Republic of Uzbekistan \\ Urgench, Uzbekistan \\ Sultanbay M. Abdikadirov ${ }^{\dagger}$ \\ Karakalpak State University \\ Nukus, Uzbekistan
}

Received 20.01.2021, received in revised form 09.02.2021, accepted 09.03.2021

\begin{abstract}
Removable singularities of separately harmonic functions are considered. More precisely, we prove harmonic continuation property of a separately harmonic function $u(x, y)$ in $D \backslash S$ to the domain $D$, when $D \subset \mathbb{R}^{n}(x) \times \mathbb{R}^{m}(y), n, m>1$ and $S$ is a closed subset of the domain $D$ with nowhere dense projections $S_{1}=\left\{x \in \mathbb{R}^{n}:(x, y) \in S\right\}$ and $S_{2}=\left\{y \in \mathbb{R}^{m}:(x, y) \in S\right\}$.
\end{abstract}

Keywords: separately harmonic function, pseudoconvex domain, Poisson integral, $\mathcal{P}$-measure.

Citation: S.A. Imomkulov, S.M. Abdikadirov, Removable Singularities of Separately Harmonic Functions, J. Sib. Fed. Univ. Math. Phys., 2021, 14(3), 369-375.

DOI: 10.17516/1997-1397-2021-14-3-369-375.

The theorem on removal of compact singularities (see [1,2]) is one of the most important results in the theory of functions in several complex variables: if a function $f$ is holomorphic everywhere in the domain $\Omega \subset \mathbb{C}^{n}(n>1)$ except a set $K \Subset \Omega$, which does not divide the domain (i.e. such that $\Omega \backslash K$ is connected), then $f$ can be extended holomorphically to whole domain $\Omega$. In the work [3], an analogue of this theorem was proved for separately harmonic functions, i.e. for functions which are harmonic in each variable separately: let $D$ be a domain in $\mathbb{R}^{n}(x) \times \mathbb{R}^{m}(y)$, $n, m>1, K \Subset D$ a compact set such that $D \backslash K$ is connected. If the function $u(x, y)$ is separately harmonic in $D \backslash K$, then it harmonically continues to $D$.

\section{Separately harmonic functions}

Definition 1. If a function $u(x, y)$ is defined in the domain $D \subset \mathbb{R}^{n}(x) \times \mathbb{R}^{m}(y)$ and satisfies the following properties:

1) for any fixed $x^{0}:\left\{x=x^{0}\right\} \cap D \neq \emptyset$, a function $u\left(x^{0}, y\right)$ is harmonic in $y$ on $\left\{x=x^{0}\right\} \cap D$;

2) for any fixed $y^{0}:\left\{y=y^{0}\right\} \cap D \neq \emptyset$, a function $u\left(x, y^{0}\right)$ is harmonic in $x$ on $\left\{y=y^{0}\right\} \cap D$, then it is called a separately harmonic function in the domain $D$.

One of the main methods of studying extension of harmonic functions is the transition to holomorphic functions, and then using the principles of holomorphic extensions.

*sevdiyor_i@mail.ru

†abdikadirov1983@inbox.ru

(c) Siberian Federal University. All rights reserved 
Lemma $1([5])$. For any domain $D \subset \mathbb{R}^{n}(x) \subset \mathbb{C}^{n}$ there is a domain of holomorphy $\widehat{D} \subset \mathbb{C}^{n}(z)$ such that $D \subset \widehat{D}$ and any harmonic function $u(x)$ in $D$ holomorphically extends into the domain $\widehat{D}$, i.e. there is a holomorphic function $f_{u}(z)$ in $\widehat{D}$ such that $\left.f_{u}\right|_{D}=u$.

The existence of the domain $\widehat{D}$ follows easily from the representation of harmonic functions by the Poisson integral. Indeed, let $B=B\left(x^{0}, R\right) \Subset D$ be an arbitrary ball in $D$, and $u(x)$ be a harmonic function in $D$. Then the following formula holds

$$
u(x)=\frac{1}{\sigma_{n}} \int_{\partial B} \frac{R^{2}-\left|x-x^{0}\right|^{2}}{R|x-y|^{n}} u(y) d \sigma(y),
$$

where $\sigma_{n}$ is the surface area of the unit sphere. It is clear that the Poisson kernel

$$
P(x, y)=\frac{1}{\sigma_{n}} \frac{R^{2}-\left|x-x^{0}\right|^{2}}{R|x-y|^{n}}
$$

for any fixed $y \in \partial B$ holomorphically extends to some domain $\widehat{B} \in \mathbb{C}^{n}, \widehat{B} \supset B$. Eventually, $\widehat{B}$ is a Lie ball centered at $x^{0}=\left(x_{1}^{0}, x_{2}^{0}, \ldots, x_{n}^{0}\right)$ with the radius $R$ (see [11])

$$
\widehat{B}=\left\{z \in \mathbb{C}^{n}: \sqrt{\left|z-x^{0}\right|^{2}+\sqrt{\left|z-x^{0}\right|^{4}-\left|\sum_{j=1}^{n}\left(z_{j}-x_{j}^{0}\right)^{2}\right|^{2}}}<R\right\} .
$$

Consequently, every harmonic in $B$ function holomorphically extends to $\widehat{B}$, which implies the existence a domain $\widehat{D}, D \subset \widehat{D} \subset \mathbb{C}^{n}$ satisfying the above properties.

It can be seen from the construction that for each fixed $z^{0} \in \widehat{D}$ there is a constant $M_{z^{0}}$ such that

$$
\left|f_{u}\left(z^{0}\right)\right| \leqslant M_{z^{0}}\|u\|_{D}
$$

nevertheless, $M_{z^{0}}$ is bounded on compact subsets of $\widehat{D}$ and

$$
\lim _{z \rightarrow x \in D} M_{z}=1
$$

\section{Separately analytic functions}

Let two domains $\mathbb{D} \subset \mathbb{C}^{n}, \mathbb{G} \subset \mathbb{C}^{m}$ and two subsets, $E \subset \mathbb{D}, F \subset \mathbb{G}$ be given. Assume that a function $f(z, w)$, determined firstly on the set $E \times F$, has the following properties:

a) for any fixed $w^{0} \in F$, a function $f\left(z, w^{0}\right)$ holomorphically extends to the domain $\mathbb{D}$;

b) for any fixed $z^{0} \in E$, a function $f\left(z^{0}, w\right)$ holomorphically extends to the domain $\mathbb{G}$.

In this case $f(z, w)$ defines some function on the set $X=(\mathbb{D} \times F) \cup(E \times \mathbb{G})$ and it is called $a$ separately-analytic function on $X$.

We will use the following theorem on analytic continuation of separately-analytic functions (V.Zakharyuta [8], J. Sichak [9], and see also [7]): let two domains $\mathbb{D} \subset \mathbb{C}^{n}, \mathbb{G} \subset \mathbb{C}^{m}$ be strongly pseudoconvex and two subsets $E \subset \mathbb{D}, F \subset \mathbb{G}$ be non-pluripolar Borel sets. If $f(z, w)$ is a separately analytic function on the set $X=(\mathbb{D} \times F) \cup(E \times \mathbb{G})$, then it extends holomorphically to the domain

$$
\widehat{X}=\left\{(z, w) \in \mathbb{D} \times \mathbb{G}: \omega^{*}(z, E, \mathbb{D})+\omega^{*}(w, F, \mathbb{D})<1\right\} .
$$


Here $\omega^{*}(z, E, \mathbb{D})$ is the $\mathcal{P}$-measure of the set $E$ with respect to the domain $\mathbb{D}$ (see $\left.[7,8,10]\right)$. It is defined as an extremal plurisubharmonic function

$$
\omega^{*}(z, E, \mathbb{D})=\varlimsup_{\zeta \rightarrow z} \omega(\zeta, E, \mathbb{D})
$$

where

$$
\omega(z, E, \mathbb{D})=\sup \left\{u(z): u \in \operatorname{psh}(\mathbb{D}),\left.u\right|_{\mathbb{D}} \leqslant 1,\left.u\right|_{E} \leqslant 0\right\}
$$

\section{On Lelong's theorem}

P. Lelong [4] proved the following analogue of the fundamental theorem of Hartogs (see [1], Ch. 1): if $u(x, y)$ is separately harmonic in the domain $D \subset \mathbb{R}^{n}(x) \times \mathbb{R}^{m}(y)$, then it is harmonic in $D$ in both variables.

The proof of Lelong's theorem can be obtained easily if we use the above theorem of V.Zakharyuta and J.Sichak: if $u(x, y)$ is separately harmonic in the domain $D \subset \mathbb{R}^{n} \times \mathbb{R}^{m}$ and $B_{1} \subset \mathbb{R}^{n}, B_{2} \subset \mathbb{R}^{m}$ are arbitrary balls such that $B_{1} \times B_{2} \subset D$, then by Lemma 1 it extends to the set $X=\left(\widehat{B}_{1} \times B_{2}\right) \cup\left(B_{1} \times \widehat{B}_{2}\right)$ as a separately analytic function. Therefore, $u(x, y)$ extends holomorphically to the domain

$$
\widehat{X}=\left\{(z, w) \in \widehat{B}_{1} \times \widehat{B}_{2}: \omega^{*}\left(z, B_{1}, \widehat{B}_{1}\right)+\omega^{*}\left(w, B_{2}, \widehat{B}_{2}\right)<1\right\} .
$$

Since $B_{1} \times B_{2} \subset \widehat{X}$, the function $u(x, y)$ is infinitely differentiable in $B_{1} \times B_{2}$ and therefore, harmonic in both variables. Since the balls are arbitrary, it follows that $u(x, y)$ is harmonic in both variables in the domain $D$.

\section{The main results}

Now we are ready to prove the main results of this paper.

Theorem 1. Let $S$ be a closed subset of the domain $D \subset \mathbb{R}^{n}(x) \times \mathbb{R}^{m}(y), n, m>1$, and its orthogonal projections $S_{1}=\left\{x \in \mathbb{R}^{n}:(x, y) \in S\right\}$ and $S_{2}=\left\{y \in \mathbb{R}^{m}:(x, y) \in S\right\}$ are nowhere dense. Then any function $u(x, y)$ which is separately harmonic in the domain $D \backslash S$ extends harmonically to the domain $D$.

Proof. Let $u(x, y)$ be a separately harmonic function in the domain $D \backslash S$ and the projections of the closed set $S$ :

$$
S_{1}=\left\{x \in \mathbb{R}^{n}:(x, y) \in S\right\}, S_{2}=\left\{y \in \mathbb{R}^{m}:(x, y) \in S\right\}
$$

are nowhere dense. We denote by $\tilde{S} \subset S$ the set of non-removable singularities for the function $u(x, y)$. Suppose that $\tilde{S} \neq \varnothing$. We take arbitrary balls $B_{1} \subset \mathbb{R}^{n}(x)$ and $B_{2} \subset \mathbb{R}^{m}(y)$ such that $B_{1} \times B_{2} \subset D$ and $\left(B_{1} \times B_{2}\right) \cap \tilde{S} \neq \varnothing$. We denote by

$$
\tilde{S}_{1}=\left\{x \in B_{1}:(x, y) \in\left(B_{1} \times B_{2}\right) \cap \tilde{S}\right\}, \tilde{S}_{2}=\left\{y \in B_{2}:(x, y) \in\left(B_{1} \times B_{2}\right) \cap \tilde{S}\right\} .
$$

Since $\left(B_{1} \times B_{2}\right) \cap \tilde{S} \subset \tilde{S}_{1} \times \tilde{S}_{2}$, we have

$$
\left(B_{1} \times B_{2}\right) \backslash\left(\tilde{S}_{1} \times \tilde{S}_{2}\right)=\left(B_{1} \times\left(B_{2} \backslash \tilde{S}_{2}\right)\right) \cup\left(\left(B_{1} \backslash \tilde{S}_{1}\right) \times B_{2}\right) \subset\left(B_{1} \times B_{2}\right) \backslash \tilde{S}
$$


Hence, by Lemma 1 , the function $u(x, y)$ can be extended analytically to the set $X=$ $=\left(\widehat{B}_{1} \times\left(B_{2} \backslash \tilde{S}_{2}\right)\right) \cup\left(\left(B_{1} \backslash \tilde{S}_{1}\right) \times \widehat{B}_{2}\right)$ as a separately analytic function. Consequently, $u(x, y)$ extends holomorphically to the domain

$$
\widehat{X}=\left\{(z, w) \in \widehat{B}_{1} \times \widehat{B}_{2}: \omega^{*}\left(z, B_{1} \backslash \tilde{S}_{1}, \widehat{B}_{1}\right)+\omega^{*}\left(w, B_{2} \backslash \tilde{S}_{2}, \widehat{B}_{2}\right)<1\right\}
$$

Since the sets $B_{1} \backslash \tilde{S}_{1}, B_{2} \backslash \tilde{S}_{2}$ are locally pluri-regular, we get

$$
X \subset \widehat{X} \text {, i.e. }\left(B_{1} \times B_{2}\right) \backslash\left(\tilde{S}_{1} \times \tilde{S}_{2}\right) \subset \widehat{X} .
$$

(About pluri-regular sets and their properties, see [6,12]). Now we take an arbitrary point $a \in \tilde{S}_{1}$ and $x^{0} \in U(a, \varepsilon) \backslash \tilde{S}_{1}$, where $U(a, \varepsilon)=\{x:|x-a|<\varepsilon\}, 0<\varepsilon<\frac{1}{2} \operatorname{dist}\left(a, \partial B_{1}\right)$. For the point $x^{0}$ there is a point $a^{0} \in \tilde{S}_{1}$ such that

$$
d=\left|x^{0}-a^{0}\right|=\inf \left\{\left|x^{0}-x\right|: x \in \tilde{S}_{1}\right\} .
$$

It is clear that the intersection $B_{1} \cap\left\{x:\left|x^{0}-x\right|<d\right\} \subset B_{1} \backslash \tilde{S}_{1}$ contains the interval $\left(x^{0}, a^{0}\right)$, which is not pluri-thin at the point $a^{0} \in \tilde{S}_{1}$ (see [6], Proposition 4.1). Hence, it follows that

$$
\omega^{*}\left(a^{0}, B_{1} \backslash \tilde{S}_{1}, \widehat{B}_{1}\right)=0
$$

On the other hand, there is a point $b^{0} \in \tilde{S}_{2}$ such that $\left(a^{0}, b^{0}\right) \in \tilde{S}$ and by the definition of $\mathcal{P}$ measure there is also some number $\delta_{2}: \omega^{*}\left(b^{0}, B_{2} \backslash \tilde{S}_{2}, \widehat{B}_{2}\right)<\delta_{2}<1$. Now we take some number $\delta_{1}>0$ so that $\delta_{1}+\delta_{2}<1$. Hence, an open neighborhood of the point

$$
\left(a^{0}, b^{0}\right) \in \tilde{S}:\left\{z: \omega^{*}\left(z, B_{1} \backslash \tilde{S}_{1}, \widehat{B}_{1}\right)<\delta_{1}\right\} \times\left\{w: \omega^{*}\left(w, B_{2} \backslash \tilde{S}_{2}, \widehat{B}_{2}\right)<\delta_{2}\right\},
$$

is contained in $\widehat{X}$, i.e. the point $\left(a^{0}, b^{0}\right) \in \tilde{S}$ is a removable singularity and this contradicts our assumption concerning $\tilde{S}$. Thus $\tilde{S}=\varnothing$. The theorem is proved.

Using methods of V. Zahariuta on analytic extension of separately analytic functions we get the following result which generalizes Hamano's theorems [3].

Theorem 2. Let two domains $D \subset \mathbb{R}^{n}, G \subset \mathbb{R}^{m}$ and two sets $E \subset D, F \subset G$ be given. If $E \Subset D$ is compact and $F$ is a closed subset of $G$ with nonempty complement $G \backslash F \neq \emptyset$, then any separately harmonic function $u(x, y)$ in $(D \times G) \backslash(E \times F)$ harmonically extends to the domain $D \times G$.

Proof. According to Lemma 1 there is a pseudoconvex domain $\widehat{G} \subset \mathbb{C}^{m}$ such that $G \subset \widehat{G}$ and for each fixed $x \in D \backslash E$ a function $u(x, \cdot)$ holomorphically extends to $\widehat{G}$. Moreover, there is a sequence of strongly pseudoconvex domains $\widehat{G}_{j}, j=1,2, \ldots$ such that $\widehat{G}_{j} \Subset \widehat{G}_{j+1} \Subset \widehat{G}$, $\widehat{G}=\bigcup_{j=1}^{\infty} \widehat{G}_{j}$ and $\left(G \cap \widehat{G}_{1}\right) \backslash F \neq \emptyset$. According to (1) for the set

$$
K_{\varepsilon}=\{z \in D: \operatorname{dist}(z, E) \leqslant \varepsilon\} \Subset D,
$$

where $\varepsilon>0$ is a small enough number, there is a sequence of positive real numbers $M_{j}$ such that

$$
|u(x, w)| \leqslant M_{j} \forall(x, w) \in \partial K_{\varepsilon} \times \widehat{G}_{j+1} .
$$


Consequently, for any $l \in N$ there is a sequence of positive numbers $N_{j}^{(l)}$ such that the inequality

$$
\sum_{|\alpha| \leqslant l}\left(\int_{\widehat{G}_{j}}\left|\frac{\partial^{|\alpha|} u(x, w)}{\partial w^{\alpha}}\right|^{2} d V\right)^{\frac{1}{2}} \leqslant N_{j}^{(l)} \forall x \in \partial K_{\varepsilon}
$$

holds.

Now we take a closed ball $\bar{B} \Subset\left(G \cap \widehat{G}_{1}\right) \backslash F$ and for a fixed $j$ and a sequence of sets $\bar{B} \Subset \widehat{G}_{j}$ we consider a Hilbert space $H_{0} \subset H_{1}$. For $H_{0}$ we take the closure of the space

$$
\mathcal{O}(\widehat{G}) \cap h(G) \cap W_{2}^{l}\left(\widehat{G}_{j}\right), l>m .
$$

(Here $\mathcal{O}(\widehat{G})$ is the space of holomorphic functions on $\widehat{G}, h(G)$ is the space of harmonic functions on $G$ and $W_{2}^{l}\left(\widehat{G}_{j}\right)$ is the Sobolev space.) For $H_{1}$ we take the closure of the space $h(G) \cap L_{2}(\bar{B}, \sigma)$, where

$$
L_{2}(\bar{B}, \mu)=\left\{f:\left(\int_{\bar{B}}|f(w)|^{2} d \sigma\right)^{\frac{1}{2}} \leqslant \infty\right\}
$$

and $d \sigma=\left(d d^{c} \omega^{*}\left(w, \bar{B}, \widehat{G}_{j}\right)\right)^{m}$ (see $\left.[7,8,10]\right)$. Let $\left\{e_{k}(w)\right\}_{k=1}^{\infty}$ be the common orthogonal basis for spaces $H_{0} \subset H_{1}$ such that $\left\|e_{k}\right\|_{H_{0}}=\mu_{k},\left\|e_{k}\right\|_{H_{1}}=1, \frac{1}{M} k^{\frac{1}{m}} \leqslant \ln \mu_{k} \leqslant M k^{\frac{1}{m}}$, and $M$ is a constant, $k=1,2, \ldots$ (see $[8,13])$.

From the continuous embedding of $H_{0} \subset C\left(\widehat{\widehat{G}}_{j}\right) \cap \mathcal{O}\left(\widehat{G}_{j}\right)$ it follows that

$$
\left|e_{k}(w)\right| \leqslant C\left\|e_{k}\right\|_{H_{0}}=C \mu_{k}, w \in \widehat{G}_{j},
$$

where $C$ is a constant.

We consider the set $A_{k}=\left\{z \in \bar{B}:\left|e_{k}(y)\right|>k\right\}$. By Chebyshev's inequality we have

$$
\sigma\left(A_{k}\right) \leqslant \frac{1}{k^{2}} \int_{\bar{B}}\left|e_{k}(y)\right|^{2} d \sigma(y)=\frac{1}{k^{2}}\left\|e_{k}\right\|_{H_{1}}=\frac{1}{k^{2}}, \quad k=1,2, \ldots
$$

Consequently, $\sum_{k=1}^{\infty} \sigma\left(A_{k}\right)<\infty$ and $\lim _{s \rightarrow \infty} \sigma\left(\bigcup_{k=s}^{\infty} A_{k}\right)=0$. We let $U_{s}=\bar{B} \backslash \bigcup_{k=s}^{\infty} A_{k}, U=\bigcup_{s=1}^{\infty} U_{s}$. Then $\sigma(\bar{B} \backslash U)=0$. Therefore, $\omega^{*}\left(w, \bar{B}, \widehat{G}_{j}\right)=\omega^{*}\left(w, U, \widehat{G}_{j}\right)$, i.e. $\omega^{*}\left(w, U_{s}, \widehat{G}_{j}\right) \downarrow \omega^{*}\left(w, \bar{B}, \widehat{G}_{j}\right)$, $w \in \widehat{G}_{j}$ (see $\left.[7,10]\right)$. Since $\left|e_{k}(y)\right| \leqslant k, w \in E_{s}, k \geqslant s$, taking into account (3), by two constants theorem we obtain the following estimation

$$
\left|e_{k}(w)\right| \leqslant c(s) k \mu_{k}^{\omega^{*}\left(w, U_{s}, \widehat{G}_{j}\right)}, \quad k \geqslant s, w \in \widehat{G}_{j},
$$

where $c(s)$ is a constant independent of $k$.

Now we compare the formal Fourier-Hartogs series to the function $u(x, w),(x, w) \subset D \times \widehat{G}_{j}$,

$$
u(x, w) \sim \sum_{k=1}^{\infty} a_{k}(x) e_{k}(w),
$$

where the coefficients are defined by the usual formulas of the space $H_{1}$ :

$$
a_{k}(x)=\int_{\bar{B}} u(x, w) \overline{e_{k}(w)} d \sigma, \quad k=1,2, \ldots
$$

We show that the series (5) converges locally uniformly in the set $K_{\varepsilon} \times \widehat{G}_{j}$. 
Since the function $u(x, y)$ is continuous and separately harmonic on the set $D \times \bar{B}$, it follows that $a_{k}(x)$ is harmonic on $D$. Moreover, for any fixed $x \in \partial K_{\epsilon}$ the function $u(x, w) \in H_{0}$, then $\left|a_{k}(x)\right|=\left(u(x, \cdot), e_{k}\right)_{H_{1}}=\mu_{k}^{-2}\left(u(x, \cdot), e_{k}\right)_{H_{0}}$. Consequently,

$$
\left|a_{k}(x)\right| \leqslant \frac{1}{\mu_{k}^{2}}\|u(x, \cdot)\|_{H_{0}}\left\|e_{k}\right\|_{H_{0}} \leqslant \frac{\|u(x, \cdot)\|_{H_{0}}}{\mu_{k}}, \quad x \in \partial K_{\varepsilon} .
$$

Hence, by the estimation (2) and the maximum principle we get the following estimation

$$
\left|a_{k}(x)\right| \leqslant \frac{N_{j}^{l}}{\mu_{k}}, k=1,2, \ldots, \quad x \in K_{\varepsilon} .
$$

Comparing the estimates (4) and (6), we obtain

$$
\left|a_{k}(x) e_{k}(w)\right| \leqslant c(s) N_{j} k \mu_{k}^{\omega^{*}\left(w, U_{s}, \widehat{G}_{j}\right)-1} \leqslant c(s) N_{j} k e^{M k^{\frac{1}{m}\left(\omega^{*}\left(w, U_{s}, \widehat{G}_{j}\right)-1\right)}},
$$

$k \geqslant s,(x, w) \in K_{\varepsilon} \times \widehat{G}_{j}$, where $U_{s} \subset \bar{B}, \sigma\left(U_{s}\right)>0$. The last estimation shows that the series (5) converges locally uniformly on the set $K_{\epsilon} \times \widehat{G}_{j}$ and its sum $\widetilde{u}(x, w)$ coincides with $u(x, w)$ on the set $\partial K_{\varepsilon} \times \widehat{G}_{j}$, i.e. $\widetilde{u}(x, w)$ is an analytic continuation of $u(x, w)$. Finally, letting $j$ tend to infinity we obtain an analytic continuation of the function $u(x, w)$ on the set $K_{\varepsilon} \times \widehat{G}$ which contains the set $E \times F$, that is the function $u(x, y)$ can be separately harmonically extended to $D \times G$. The proof of Theorem 2 is completed.

Comparing the ideas of proof of theorems above, one can easily prove the following theorem:

Theorem 3. Let two domains $D \subset \mathbb{R}^{n}, G \subset \mathbb{R}^{m}$ and two sets $E \subset D, F \subset G$ be given. If $E$ is a nowhere dense closed subset of the domain $D$ and $F$ is a closed subset of the domain $G$ with a non-empty complement $G \backslash F \neq \emptyset$, then any separately harmonic function $u(x, y)$ on the domain $(D \times G) \backslash(E \times F)$ can be extended harmonically to the domain $D \times G$.

\section{References}

[1] B.V.Shabat, Introduction to Complex Analysis, Part II, Moscow, Nauka, Fiz. Mat. Lit., 1985 (in Russian).

[2] R.Gunning, H.Rossi, Analytic functions of several complex variables, Chelsea Publishing, American Mathematical Society, Providence, Rhode Island, 1965.

[3] S. Hamano, Hartogs-Osgud theorem for separately harmonic functions, Proc. Japan Acad. $A, \mathbf{8 3}(2007), 16-18$. DOI: $10.3792 /$ pjaa.83.16

[4] P.Lelong, Fonctions plurisousharmoniques et fonctions analytiques de variables reelles, Ann. Inst. Fourier. Paris., 11(1961), 515-562.

[5] A.Sadullaev, S.A.Imomkulov, Extension of holomorphic and pluriharmonic functions with subtle singularities on parallel sections, Proc. Steklov Inst. Math., 253(2006), 144-159.

DOI: $10.1134 /$ S0081543806020131

[6] A.Sadullaev, Plurisubharmonic measures and capacities on complex manifolds, UMN, 36(4)(1981), 53-105.

[7] A.Sadullaev, Plurisubharmonic functions, Itogi Nauki i Tekhniki. Ser. Sovrem. Probl. Mat. Fund. Napr., 8(1985), 65-111 (in Russian). 
[8] V.Zakharyuta, Separate-analytic functions, generalized Hartogs theorems and hulls of holomorphy, Math. Coll., 101(1976), no. 1, 57-76.

[9] J.Siciiak, Separately analytic functions and envelopes of holomorphy of some lowerdimensional subsets of $\mathbb{C}^{n}$, Ann. Pol. Math., 22(1969), no, 1, 145-171.

[10] E.Bedford, B.A.Taylor, A new capacity for plurisubharmonic functions, Acta. Math., 149(1982), 1-40.

[11] V.Avanissian, Cellule d'Harmonicite et Prolongement Analitique Complexe, Travaux en cours, Hermann, Paris, 1985.

[12] W.Plesniak, A criterion of the L-regulerity of compact sets in $\mathbb{C}^{n}$, Zeszyty Neuk. Uniw. Jagiello., 21(1979), 97-103.

[13] B.Mityagin, Approximate dimension and bases in nuclear spaces, Russian Math. Surveys, 16(1961), no, 4, 59-127.

\title{
Стираемые особенности сепаратно-гармонических функций
}

Севдиёр А. Имомкулов

Хорезмский областной филиал Математического института им. В. И. Романовского

Академия наук Республики Узбекистан

Ургенч, Узбекистан

Султанбай М. Абдикадиров

Каракалпакский государственный университет

Нукус, Узбекистан

\begin{abstract}
Аннотация. В работе рассматриваются устранимые особенности сепаратно-гармонических функций. Точнее, доказана теорема о гармоническом продолжении сеператно-гармонической в $D \backslash S$ функции $u(x, y)$ в область $D$, где $D \subset \mathbb{R}^{n}(x) \times \mathbb{R}^{m}(y), n, m>1$ и $S$ - замкнутое подмножество области $D$, а ее проекции $S_{1}=\left\{x \in \mathbb{R}^{n}:(x, y) \in S\right\}$ и $S_{2}=\left\{y \in \mathbb{R}^{m}:(x, y) \in S\right\}$ нигде не плотны.

Ключевые слова: сепаратно-гармоническая функция, псевдовыпуклая область, интеграл Пуассона, $\mathcal{P}$-мера.
\end{abstract}

